\title{
Nonlinear Identification of a Solar Heating System
}

\author{
Linda Brus \\ Systems and Control, Department of Information Technology, Uppsala University \\ P.O. Box 337, SE-751 05 Uppsala, SWEDEN
}

\begin{abstract}
The use of solar heating systems is a way of exploiting the clean and free energy from the sun. To optimize the energy gain from such a system, where the main input, the solar insolation, is an uncontrollable variable, good models of the system dynamics are required. Identification methods are often either highly specialized for the application or require an extensive amount of data, especially when the dynamics studied are nonlinear. This paper shows that by application of a new recursive system identification technique, a small scale solar heating system can be modeled with very little data, without having to tailor the model structure to the application.
\end{abstract}

\section{INTRODUCTION}

The sun is the primary energy source of the earth, providing the planet with light and heat. The energy from the sun is a lasting environmentally friendly resource that leaves no waste products, and it is available in amounts that greatly exceeds our demands. As compared to the use of fossil fuels, the long term advantages appear to be overwhelming. The main problem with solar energy is, however, how to utilize, store, and distribute it. Heating of buildings and hot water is one common way to make use of the solar energy.

In this paper a small scale solar heating system is modeled with nonlinear identification. The system consists of a solar collector, a heat storage, and a fan that blows hot air from the former to the latter. The heat can then be distributed from the heat storage to the house. The system is used for building heating only.

Nonlinearities are not uncommon in solar energy applications, which poses a problem in modeling and control of e.g. solar collector fields. Good control strategies are crucial to obtain efficient utilization of the solar energy. For example, the energy loss from the solar collectors increases as the temperature increases, which calls for low influent temperature, so that the energy loss is minimized. At the same time the objective of a solar heating system is to utilize as much energy as possible and thereby increasing the temperature in the collectors. Examples of efforts to increase the energy gain from solar collectors, by the use of nonlinear models include [1], [2], and [3]. In [1] an adaptive nonlinear controller of a solar collector field is designed, [2] describes a study of energy based control of solar collectors, and [3] uses neural identification methods to obtain a model for predictive controller design.

With accurate models of the solar heating process available, controller design can be facilitated, and the energy gain from the collector improved. However, if the accuracy of the model is obtained at the cost of high complexity and many model parameters, the controller design may be complicated.
The ideal would be a simple model structure that gives a high accuracy. Such a model could be used for various applications, like fault detection and weather forecast based control. Note that weather forecast based control would rely on a dynamic model of the energy utilization, since only predicted solar insolation is available for present control decisions.

Given the background described above a first objective of the paper is to apply a new nonlinear system identification technique to measured data from the solar heating system. At the same time, this provides a benchmark for the new recursive algorithm. A second objective is to study the obtainable performance when the data set is very small and the main input signal excitation (solar insolation) is limited. The intention is, naturally, to investigate the practical feasibility of on-line nonlinear modeling, to prepare for nonlinear optimal control, e.g. utilizing weather forecasts.

Today linear system identification has been widely studied and is well developed, and in many cases linear models will be sufficient to describe the behavior of a system. There are also several methods to solve nonlinear identification problems, but due to the complexity of the subject, this area is not as thoroughly studied as the linear identification methods. Previous studies of nonlinear identification strategies to different engineering applications include power plant modeling [4], pulp digester modeling and control [5], and modeling of closed loop plant processes [6] just to mention a few.

Identification methods are often classified as grey-box (semi-physical), or black-box (non-physical) models. In greybox modeling a priori knowledge of the system dynamics is included in the model structure, followed by estimation of a number of unknown model parameters using measured data. Examples of grey-box modeling include [7] and [8]. In black-box identification no previous knowledge of the system is required, which can be an advantage if information of the system dynamics is limited, but it also involves the problem of choosing a suitable model structure.

A recursive prediction error method (RPEM) for solving nonlinear identification problems with a restricted blackbox parameterization was described in [9], [10]. The model involves a multi-variable polynomial, which offers flexibility in terms of application areas. In this paper the RPEM is applied to a limited data set from the small scale solar heating system outlined above.

This paper is organized as follows. In section II the solar heating system is described. Section III presents the model 


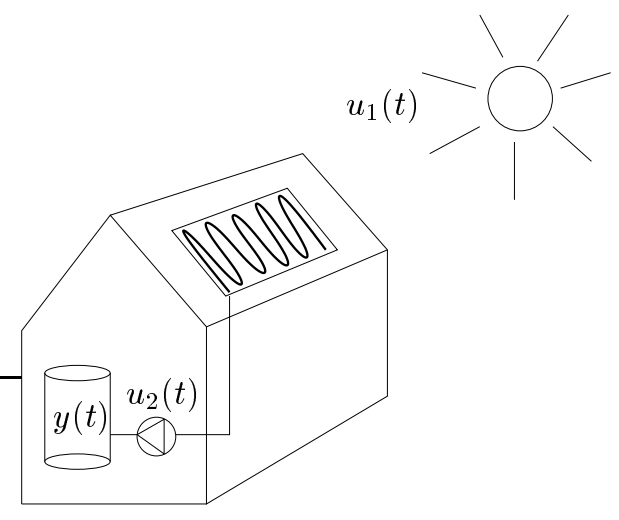

Fig. 1. A schematic picture of the solar heating system, with the measured variables irradiance (solar insolation), $u_{1}(t)$, fan switch state, $u_{2}(t)$, and heat storage temperature, $y(t)$.

parameterization, followed by experiments in section IV. Finally, the conclusions can be found in section V.

\section{SySTEM DESCRIPTION}

The main component of a solar heating system is the solar collector. Depending on application and locality of the system the collector can differ in design, but common to all solar collectors is that they are constructed to absorb the solar radiation and transfer it to heat. The heat is then transported by some kind of medium to a heat storage. The medium can be air, but more common is to use a fluid, e.g. oil or a water and anti-freeze mixture.

The energy gain from the solar collector is determined by the balance between absorbed energy and heat loss to the environment. The heat loss increases with temperature and limits the maximum temperature attained from the system. To optimize the amount of extracted energy from the system it is therefore of interest to minimize the temperature loss from the collector. Hence, the ideal collector giving maximum temperatures and maximum useful power outputs is highly absorbent, well-insulated, and exposed to intense solar radiation.

The data [11] used for the experiments are from a small scale solar heating system, see Fig. 1. The collector, which has air as energy transportation medium, is connected to a heat storage filled with pebbles that retain the energy from the collector. There is also a fan, which controls the air flow between the collector and the heat storage. Data consist of measurement of solar insolation, $u_{1}(t)$, fan switch state (on/off), $u_{2}(t)$, and the temperature of the heat storage, $y(t)$, during approximately two days, see Fig. 2. The sampling period is 10 minutes and the total number of samples is 296 .

Several different off-line modeling approaches have previously been applied to this data set. In [12] a linear model, a neural network approach, and a grey-box model that involves reparameterization are presented.

The amount of data is limited, and for many nonlinear black-box identification methods 296 samples may not be
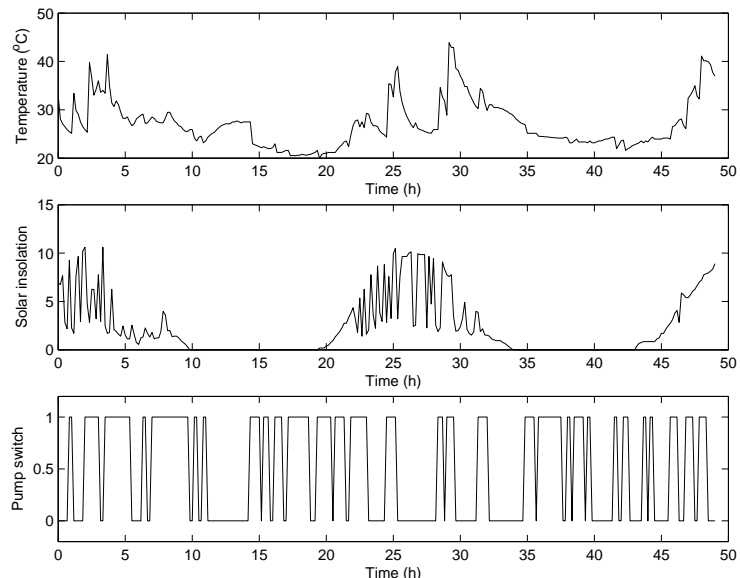

Fig. 2. Measured data from the solar heating system, heat storage temperature, $y(t)$ (top), solar insolation, $u_{1}(t)$ (middle), and fan switch state, $u_{2}(t)$ (bottom).

sufficient to obtain an accurate model. However, this paper aims to show that the proposed black-box recursive algorithm can produce highly accurate models even with a small data set, by multiple scans of the algorithm over the data set. Note that only 148 samples (50\%) are used for identification, the second half of the data set is used for validation.

\section{MOdEL AND ALGORITHM}

\section{A. Model}

The identification was performed with a recursive prediction error algorithm with a restricted black-box parameterization. To describe the identification model, which is of output error (OE) type, introduce the input vector

$$
\boldsymbol{u}(t)=\left(u_{1}(t) \ldots u_{1}^{\left(n_{1}\right)}(t) \ldots u_{k}(t) \ldots u_{k}^{\left(n_{k}\right)}(t)\right)^{T}
$$

and the state vector

$$
\boldsymbol{x}(t)=\left(\begin{array}{llll}
x_{1}(t) & x_{2}(t) \quad \ldots \quad x_{n}(t)
\end{array}\right)^{T}
$$

The superscript $j$ of $u_{i}^{(j)}$ denotes the $j^{t h}$ derivative of the $i^{t h}$ input signal. The algorithm is based on the state space model

$$
\begin{aligned}
& \boldsymbol{x}^{(1)}=\left(\begin{array}{c}
x_{1}^{(1)} \\
\vdots \\
x_{n-1}^{(1)} \\
x_{n}^{(1)}
\end{array}\right)=\left(\begin{array}{c}
x_{2} \\
\vdots \\
x_{n} \\
f(\boldsymbol{x}, \boldsymbol{u}, \boldsymbol{\theta})
\end{array}\right) \\
& y(t)=\left(\begin{array}{llll}
1 & 0 & \ldots & 0
\end{array}\right) \boldsymbol{x}
\end{aligned}
$$

where $\boldsymbol{\theta}$ is the unknown parameter vector containing $n_{\theta}$ components. The right-hand side of (4) can be exchanged for any set of known nonlinear vector functions to expand the model to a general multiple output model (cf. [9]).

By limiting the nonlinearity to entering equation (3) in one of the right-hand side components only, overparameterization can be avoided and the model structure complexity kept relatively low. Yet, the state space form (3)-(4) provides a possibility to model a wide variety of right-hand side components through the function $f$. In fact, it is proven in 
[9] and the references therein that the model can (locally) describe systems with arbitrary right-hand side structures.

$f(\boldsymbol{x}, \boldsymbol{u}, \boldsymbol{\theta})$ is chosen to be of polynomial form

$$
\begin{aligned}
& f(\boldsymbol{x}, \boldsymbol{u}, \boldsymbol{\theta})= \\
& =\sum_{i_{x_{1}}=0}^{I_{x_{1}}} \ldots \sum_{i_{x_{n}}=0}^{I_{x_{n}}} \sum_{i_{u_{1}}=0}^{I_{u_{1}}} \ldots \sum_{i_{u_{1}^{\left(n_{1}\right)}}=0}^{I_{u_{1}^{\left(n_{1}\right)}}} \ldots \sum_{i_{u_{k}}=0}^{I_{u_{k}}} \ldots \sum_{i_{u_{k}}^{\left(n_{k}\right)}=0}^{I_{u_{k}\left(n_{k}\right)}^{I_{k}}}
\end{aligned}
$$

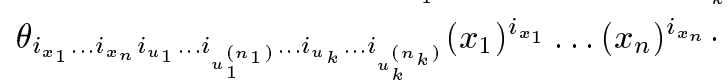

$$
\begin{aligned}
& \cdot\left(u_{1}\right)^{i_{u_{1}}} \ldots\left(u_{1}^{\left(n_{1}\right)}\right)^{i u_{1}^{\left(n_{1}\right)}} \ldots\left(u_{k}\right)^{i_{u_{k}}} \ldots\left(u_{k}^{\left(n_{k}\right)}\right)^{i u_{k}^{\left(n_{k}\right)}}= \\
& =\varphi^{T}(\boldsymbol{x}, \boldsymbol{u}) \boldsymbol{\theta}
\end{aligned}
$$

For example, a simple first order model of this representation would be with one state, $x$ (corresponding to the output signal, cf. equation (4)) and two input signals, $u_{1}$ and $u_{2}$ where $n_{1}=n_{2}=0$ (the differential equation does not depend on derivatives of the input signals in this case). This means that $n_{\theta}=8$,

$$
\boldsymbol{\theta}=\left(\begin{array}{llllllll}
\theta_{000} & \theta_{001} & \theta_{010} & \theta_{011} & \theta_{100} & \theta_{101} & \theta_{110} & \theta_{111}
\end{array}\right)^{T}
$$

and

$$
\varphi=\left(\begin{array}{llllllll}
1 & u_{2} & u_{1} & u_{1} u_{2} & x & x u_{2} & x u_{1} & x u_{1} u_{2}
\end{array}\right)^{T}
$$

Remark 1: A discussion on the consequences of the use of input signal derivatives appears in [9]. Note that no input signal derivatives are used in this paper.

\section{B. Discretization}

To be able to formulate an RPEM algorithm the model needs to be discretized. Applying the Euler integration method to (3), and using the relations (4) and (5) the following discrete time model is obtained

$$
\begin{gathered}
\left(\begin{array}{c}
x_{1}\left(t+T_{S}, \boldsymbol{\theta}\right) \\
\vdots \\
x_{n-1}\left(t+T_{S}, \boldsymbol{\theta}\right) \\
x_{n}\left(t+T_{S}, \boldsymbol{\theta}\right)
\end{array}\right)= \\
\left(\begin{array}{c}
x_{1}(t, \boldsymbol{\theta}) \\
\vdots \\
x_{n-1}(t, \boldsymbol{\theta}) \\
x_{n}(t, \boldsymbol{\theta})
\end{array}\right)+T_{S}\left(\begin{array}{c}
x_{2}(t, \boldsymbol{\theta}) \\
\vdots \\
x_{n}(\boldsymbol{\theta}) \\
\boldsymbol{\varphi}^{T}(\boldsymbol{x}, \boldsymbol{u}, \boldsymbol{\theta}) \boldsymbol{\theta}
\end{array}\right) \\
y(t, \boldsymbol{\theta})=\left(\begin{array}{llll}
1 & 0 & \ldots & 0) \boldsymbol{x}(t, \boldsymbol{\theta})
\end{array}\right.
\end{gathered}
$$

\section{RPEM}

From the discretized model an RPEM can now be formulated. The identification is performed using an output error approach, and the covariance matrix of the measurement disturbances is estimated on-line. The construction of the algorithm follows the standard approach of [13]. The RPEM is given by

$$
\begin{aligned}
& \varepsilon(t)=y_{m}(t)-y(t) \\
& \boldsymbol{\Lambda}(t)=\boldsymbol{\Lambda}\left(t-T_{S}\right)+\frac{\mu(t)}{t}\left(\varepsilon(t) \varepsilon^{T}(t)-\boldsymbol{\Lambda}\left(t-T_{S}\right)\right) \\
& \boldsymbol{R}(t)=\boldsymbol{R}\left(t-T_{S}\right)+\frac{\mu(t)}{t}\left(\boldsymbol{\psi}(t) \boldsymbol{\Lambda}^{-1}(t) \boldsymbol{\psi}^{T}(t)-\boldsymbol{R}\left(t-T_{S}\right)\right) \\
& \hat{\boldsymbol{\theta}}(t)=\left[\hat{\boldsymbol{\theta}}\left(t-T_{S}\right)+\frac{\mu(t)}{t} \boldsymbol{R}^{-1}(t) \boldsymbol{\psi}(t) \boldsymbol{\Lambda}^{-1}(t) \varepsilon(t)\right]_{D_{M}} \\
& \left(\begin{array}{c}
x_{1}\left(t+T_{S}\right) \\
\vdots \\
x_{n-1}\left(t+T_{S}\right) \\
x_{n}\left(t+T_{S}\right)
\end{array}\right)= \\
& =\left(\begin{array}{c}
x_{1}(t) \\
\vdots \\
x_{n-1}(t) \\
x_{n}(t)
\end{array}\right)+\alpha T_{S}\left(\begin{array}{c}
x_{2}(t) \\
\vdots \\
x_{n}(t) \\
\varphi^{T}(t) \hat{\boldsymbol{\theta}}(t)
\end{array}\right) \\
& \boldsymbol{y}\left(t+T_{S}\right)=\left(\begin{array}{llll}
1 & 0 & \ldots & 0
\end{array}\right) \boldsymbol{x}\left(t+T_{S}\right) \\
& \frac{d \varphi}{d x_{i}}(t)=\left(\mathbf { 0 } ^ { T } 1 u _ { k } ^ { ( n _ { k } ) } ( t ) \ldots \left(\left(x_{i+1}(t)\right)^{I_{x_{i+1}}} \ldots\right.\right. \\
& \ldots\left(x_{n}(t)\right)^{I_{x_{n}}}\left(u_{1}(t)\right)^{I_{u_{1}}} \ldots\left(u_{k}^{\left(n_{k}\right)}(t)\right)^{I_{u_{k}}^{\left(n_{k}\right)}} \\
& \left.\left.2 x_{i}(t) \quad 2 x_{i}(t) u_{k}^{\left(n_{k}\right)}(t) \ldots\right)\right), \quad i=1, \ldots, n \\
& \frac{d \varphi}{d x}(t)=\left(\begin{array}{c}
\frac{d \varphi}{d x_{1}}(t) \\
\vdots \\
\frac{d \varphi}{d x_{n}}(t)
\end{array}\right) \\
& \left(\begin{array}{c}
\frac{d x_{1}}{d \boldsymbol{\theta}}\left(t+T_{S}\right) \\
\vdots \\
\frac{d x_{n-1}}{d \boldsymbol{\theta}}\left(t+T_{S}\right) \\
\frac{d x_{n}}{d \boldsymbol{\theta}}\left(t+T_{S}\right)
\end{array}\right)=\left(\begin{array}{c}
\frac{d x_{1}}{d \boldsymbol{\theta}}(t) \\
\vdots \\
\frac{d x_{n-1}-1}{d \boldsymbol{\theta}}(t) \\
\frac{d x_{n}}{d \boldsymbol{\theta}}(t)
\end{array}\right)+\alpha T_{S} \\
& \left(\begin{array}{c}
\frac{d x_{2}}{d \boldsymbol{\theta}}(t) \\
\vdots \\
\left.\left.\varphi^{T}(t)+\hat{\boldsymbol{\theta}}(t)\left(\frac{d \varphi}{d \boldsymbol{x}}(t)\right)\right)^{\frac{d x_{n}}{d \boldsymbol{\theta}}(t)}\left(\frac{d x_{1}}{d \boldsymbol{\theta}}(t)\right)^{T} \ldots\left(\frac{d x_{n}}{d \boldsymbol{\theta}}(t)\right)^{T}\right)
\end{array}\right) \\
& \boldsymbol{\psi}\left(t+T_{S}\right)=\left(\begin{array}{llll}
1 & 0 & \ldots & 0
\end{array}\right) \frac{d \boldsymbol{x}}{d \boldsymbol{\theta}}\left(t+T_{S}\right)
\end{aligned}
$$

Here $\varepsilon(t)$ is the prediction error, $\boldsymbol{\Lambda}(t)$ is the running estimate of the covariance matrix of the measurement disturbance, $\frac{\mu(t)}{t}$ is the gain sequence, $\boldsymbol{R}(t)$ is the running estimate of the Hessian, and $\psi(t)$ is the gradient of the output prediction with respect to the parameter vector. The gradient is determined by dynamic recursion, using the dynamics from the linearized state space model of the system. $D_{M}$ is the set of parameter estimates that give stable models, and is introduced to ensure model stability. $D_{M}$ is determined by linearization. $\alpha$ is a scaling factor applied to the sampling period, see [10] for further details.

\section{Model characteristics and algorithmic properties}

The main advantage of the model used in this paper is that it enables a black-box approach for a nonlinear identification 
problem. The restricted black-box parameterization of the state space ODE, with a polynomial model of one right-hand side of the ODE, prevents overparameterization. In addition the polynomial can, locally, model more complicated nonlinearities in the right-hand side of the ODE [9]. Due to the generality of a black-box approach, the model could be used for various applications within many engineering fields.

Certain conditions may, however, cause problems with the identification. For example, if the relative sizes of the state vector components differ a lot, the polynomial elements will differ in magnitude too. This may cause numerical problems in the RPEM algorithm. The scaling factor $\alpha$ in (10) can be applied to the sampling period to improve the conditioning of the identification problem, see [10] for further details.

Abnormal behavior may occur e.g. when the parameter values leave the stability region. When this happens the parameter estimates will be changed back to their values from the previous time step. From here the algorithm may take the same route again, and hence the parameter estimates may be stuck on the margin of the stability region.

\section{EXPERIMENTS}

\section{A. Application and data}

As mentioned previously the data from the solar heating system consists of two inputs and an output. The solar insolation $u_{1}$ varies over the day, and during the night there are, naturally, long periods of no solar radiation. This introduces some difficulties, since there is no excitation of $u_{1}$ during this time. The second output $u_{2}$ corresponds to the speed of the fan transporting warm air from the solar collector to the heat storage. The fan has one speed only, hence $u_{2}$ is a binary signal where $1 / 0$ means on/off. Thirdly, the output signal $y$ represents the air temperature in the heat storage, measured at the inlet from the solar collector.

To be able to compare model performances the data set was split in two, one subset for calibration of the model parameters and a second for validation of the calibrated model. The calibration set consists of the first half of the samples and the validation data of the second half.

\section{B. Small data set - multiple scans}

As mentioned earlier, the data set used for calibration only contains 148 samples. From a modeling perspective this is a small data set, especially when considering that the identification method is of black-box type. In fact, 150 data points is unlikely to be enough for the algorithm to converge from an arbitrary set of initial parameters. To avoid this problem multiple scans have been introduced.

The idea behind the multiple scans is to apply the algorithm to the data set several times, each time changing the initial parameters to the final parameters of the previous iteration. This way an arbitrary set of initial parameters could eventually converge to a minimum, even for a small data set. An acceptable model could be obtained from a small amount of data even if the initial guess is poor. If the data set would have been larger the multiple scans would be superfluous. Note that multiple scans are only believed to be required during initial convergence. When tracking the model on-line the RPEM is believed to be able to cope with changes of dynamics, as reflected by a few tens of samples. See below for further comments.

The nonlinear model structure increases the risk of instability issues and convergence to local minima significantly. There is no stringent way of determining if a model is in fact the best model obtainable, or if it is just a local minimum. Similarly, in case the algorithm fails to converge to a good model, it is difficult to know whether this is because it lacks the required polynomial elements, or if it is a result of e.g. badly chosen initial parameters. However, it may not be crucial to find the optimal model, but rather one with a model performance satisfactory for the intended application.

\section{Identification results}

Identification experiments with the solar heating system data have been performed using the model described by equations (3)-(7) through the RPEM (10). The experiments were of black-box type, where no prior knowledge of the system was assumed to be available. The starting point was then to use the whole $\varphi$ and $\theta$ vectors described in (6) and (7), and then exclude one polynomial element at a time to determine which parameters improve the model performance the most.

The algorithm was initialized with

$$
\theta_{0}=\left[\begin{array}{llllllll}
0 & 5 & 0.5 & 0 & -0.08 & 0 & 0 & 0
\end{array}\right]^{T}
$$

A comparison to (6) shows that all the initial nonlinear parameters have been chosen to zero. The first element, corresponding to the bias parameter, was also chosen to be zero. The initial bias parameter did, however, cause difficulties. The estimation of the bias parameter totally dominated the identification procedure, leaving the static gains (element 2 and 3) relatively unchanged. This lead to convergence to a local minimum that resulted in a poor model. To solve this problem the bias parameter of $\theta_{0}$ was excluded until the static gains had adapted, after which it was added to the parameter vector again. The model obtained by this procedure was significantly better than the one obtained when just using (11).

Each of the other, reduced, models were obtained by removing one parameter at the time from the full eight parameter model. For some of the parameter combinations the estimates changed a lot over the calibration data set, resulting in bad models. The rapid changes in parameter estimates, see Fig. 3, indicated that the parameter updating gain, $\frac{\mu(t)}{t} R^{-1}(t) \psi(t) \Lambda^{-1}(t)$, was too high. This can be avoided by increasing the initial value of the Hessian, $R$, or reducing the size of the gain sequence $\frac{\mu(t)}{t}$. By the same reasoning reducing the Hessian or increasing the gain sequence increase the changes in the parameter estimates, which can be desirable e.g. if the algorithm does not adapt fast enough or converges to a local minimum.

Examples of simulations with models obtained in the experiments are shown in Fig. 4 and 5. In Table I the mean 
TABLE I

EXPERIMENTS. THE LEFT COLUMN INDICATES WHICH PARAMETERS ARE NOT INCLUDED IN THE MODEL. THE RIGHT COLUMN CONTAINS THE MEAN SQUARE ERROR AFTER CONVERGENCE.

\begin{tabular}{|l||c|}
\hline Excluded parameters & Mean Square Error \\
\hline None (full model) & 15.0 \\
$\theta_{000}$ & 17.0 \\
$\theta_{001}$ & 15.5 \\
$\theta_{010}$ & 15.7 \\
$\theta_{011}$ & 14.7 \\
$\theta_{100}$ & 15.1 \\
$\theta_{101}$ & 19.1 \\
$\theta_{110}$ & 14.6 \\
$\theta_{111}$ & 15.6 \\
$\theta_{010}, \theta_{011}$ & 11.2 \\
$\theta_{010}, \theta_{110}$ & 12.1 \\
$\theta_{011}, \theta_{110}$ & 11.3 \\
$\theta_{010}, \theta_{011}, \theta_{110}$ & 11.9 \\
$\theta_{010}, \theta_{110}, \theta_{111}$ & 11.6 \\
$\theta_{010}, \theta_{011}, \theta_{100}, \theta_{110}$ & 46.3 \\
\hline
\end{tabular}

square error

$$
M S E=\frac{1}{N} \sum_{t}\left(y_{m}(t)-y(t)\right)^{2}
$$

of the simulated output with each model are presented.

The model performance was only improved when one of the parameters $\theta_{011}$ and $\theta_{110}$, was removed, all other parameter removals resulted in a higher MSE after convergence. With $\theta_{010}$ removed, however, convergence was obtained after increasing the Hessian by a factor 3 . With the original Hessian the algorithm reached a point where it alternated between two parameter estimates, one giving an MSE of 20.1 and the other 13.4. The lower MSE suggests that removal of $\theta_{010}$ may lead to improved model performance, even though the converged parameter set did not indicate that. The experiment was therefore extended to removing also different combinations of $\theta_{010}, \theta_{010}$, and $\theta_{110}$. As Table I shows, removal of any combination of two of these three parameters improves the model significantly. Removal of all three parameters also results in a relatively good model.

The original removal of only $\theta_{100}$ did not seem to affect the model behavior significantly. However, an attempt to remove the $\theta_{100}$ parameter from the model where $\theta_{010}, \theta_{011}$ and $\theta_{110}$ had been removed resulted in a very poor model.

As an extra experiment $\theta_{011}$ was exchanged for $\theta_{111}$ (in the model with three removed parameters) under the hypothesis that the important aspect would be to have a term in the model containing the product of the two inputs, but that it would be of lesser importance whether it was $u_{1} u_{2}$ or $x u_{1} u_{2}$. The hypothesis was supported by the identification results, the MSE was even slightly lower for the model where $\theta_{111}$ was removed, compared to that where $\theta_{011}$ was removed.

Finally, a case when the whole data set was used for identification is displayed in Fig. 6. The simulation was

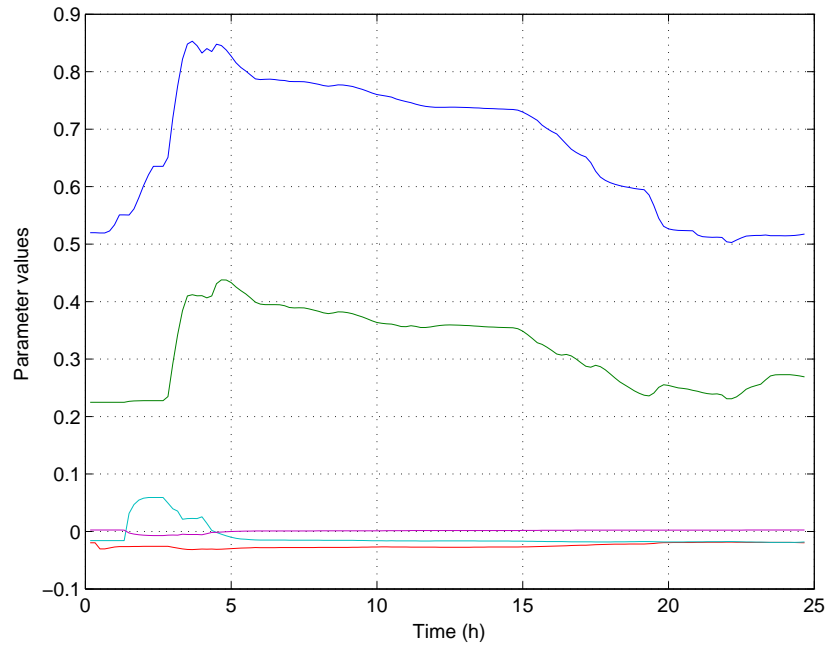

Fig. 3. Parameter estimations for the model where $\theta_{010}$ and $\theta_{011}$ has been excluded. The initial Hessian is here equal to the identity matrix of dimension $n_{\theta}\left(R_{0}=I_{n_{\theta}}\right)$

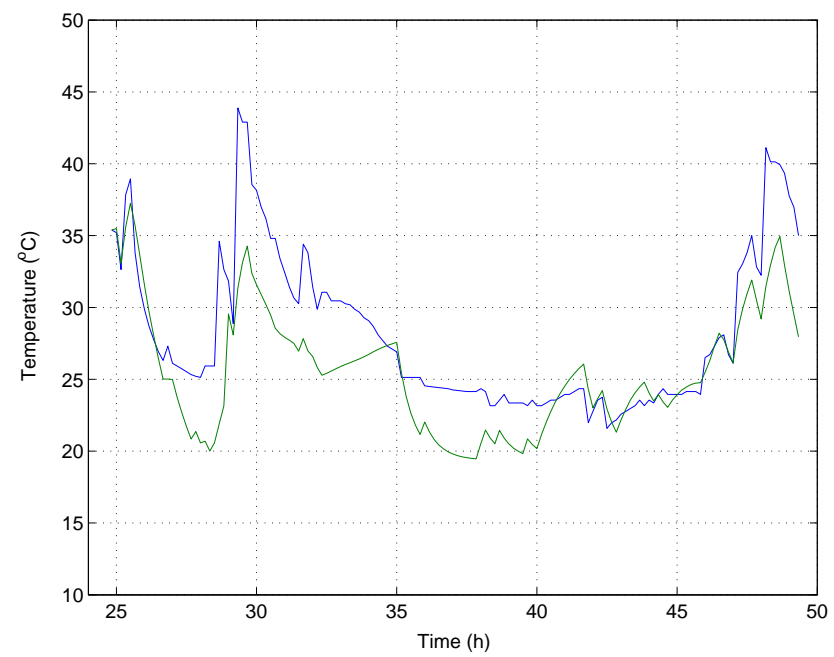

Fig. 4. Measured (blue) and simulated (green) heat storage temperature for the full black-box model.

also done over the entire data set, using the final parameter estimate from the identification.

\section{Comparison with an alternative model}

Since the same data set has been treated previously by [12] a comparison of model performance would be interesting. Hence, the off-line reparameterization experiment presented by Ljung and Glad has been performed on the same calibration and validation data sets to enable a comparison. The reparameterized model,

$$
\begin{aligned}
y(t) & =\theta^{T}(t) \varphi(t) \\
\varphi(t) & =\left[\begin{array}{llll}
\varphi_{1}(t) & \varphi_{2}(t) & \ldots & \varphi_{6}(t)
\end{array}\right]^{T}
\end{aligned}
$$




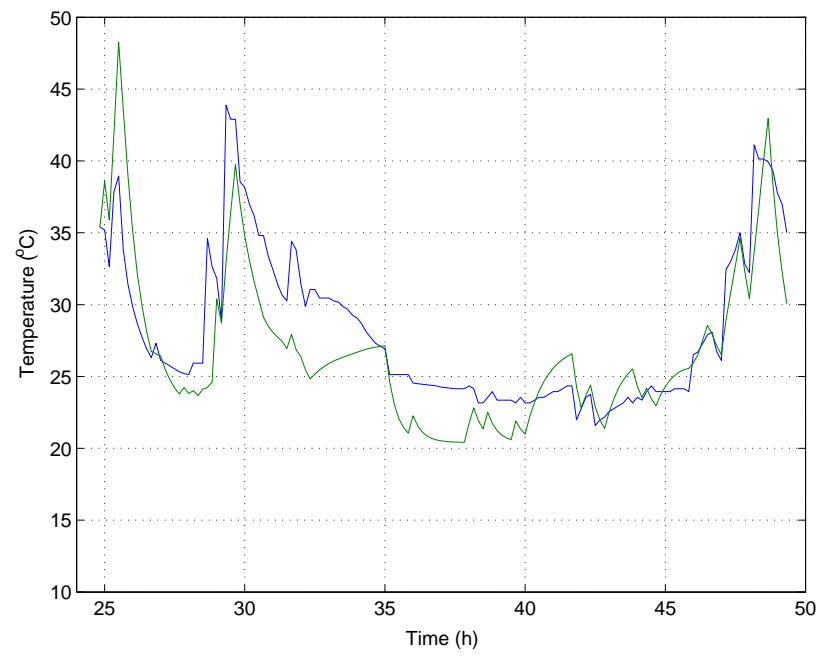

Fig. 5. Measured (blue) and simulated (green) heat storage temperature for the model where $\theta_{010}$ and $\theta_{011}$ has been excluded.

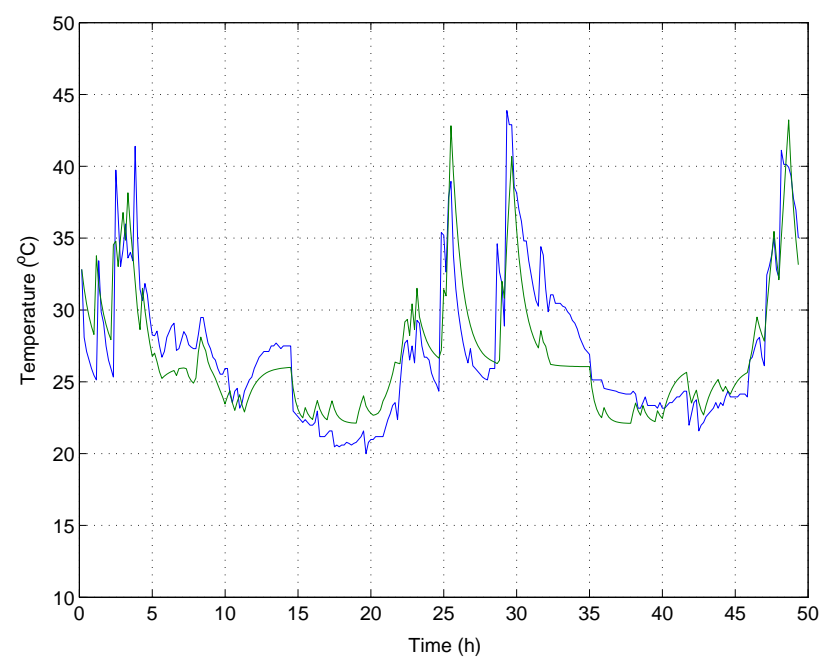

Fig. 6. Measured (blue) and simulated (green) heat storage temperature for the model where $\theta_{010}, \theta_{011}$, and $\theta_{110}$ has been excluded. The whole data set has been used for identification.

where

$$
\begin{aligned}
& \varphi_{1}(t)=y(t-1) \\
& \varphi_{2}(t)=y(t-1) u_{2}(t-1) / u_{2}(t-2) \\
& \varphi_{3}(t)=y(t-2) u_{2}(t-1) / u_{2}(t-2) \\
& \varphi_{4}(t)=u_{2}(t-1) u_{1}(t-2) \\
& \varphi_{5}(t)=y(t-1) u_{2}(t-1) \\
& \varphi_{6}(t)=u_{2}(t-1) y(t-2)
\end{aligned}
$$

is based on an energy balance of the solar heating system.

As in the RPEM model the off-line model has parameters that are more crucial to the model performance than others. In both cases there were also a substantial improvement when some of the more superfluous parameters were excluded from the model. Hence, the best model obtained with the RPEM model has been compared to a reduced version of (13)-(14), containing the parameters $\varphi_{1}(t), \varphi_{2}(t), \varphi_{3}(t), \varphi_{4}(t)$, and

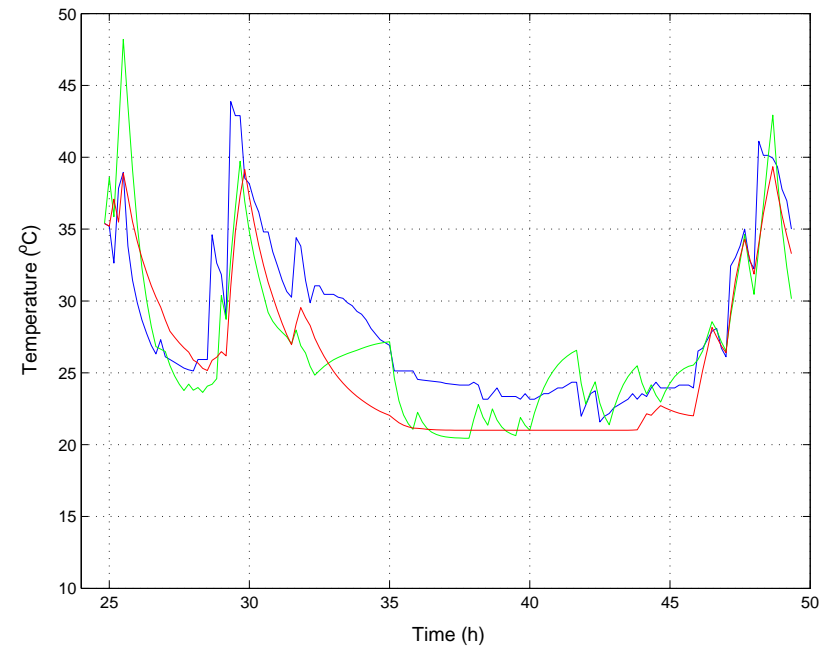

Fig. 7. Measured temperature of the heat storage (blue), as well as simulated ditto; the black-box model where $\theta_{010}$ and $\theta_{011}$ has been excluded (green), and a reduced version of the reparameterized model presented in [12] (red).

$\varphi_{6}(t)$. The reparameterization method also requires removal of background temperature before the identification event. In this case the background temperature has been assumed to be $21^{\circ} \mathrm{C}$. It might have been possible to reduce the MSE of the off-line model further by subtracting an other background temperature (perhaps time varying) or by using a different subset of parameters. The results appear in Fig. 7.

Some comments are in order here. Note that the model (13)-(14) settles to a constant output during periods with zero solar insolation. This is because only the product of $u_{1}$ and $u_{2}$ affects the output signal. A consequence is that this model underestimates the temperature during night. The black-box RPEM, however, retains a direct impact from the fan $\left(u_{2}\right)$ on the output, a fact which allows the model to compensate somewhat during periods of zero solar insolation. This is why the output of the RPEM model does not settle to a constant value during periods when $u_{1}=0$.

The MSE of the RPEM and reparameterization methods were 11.2 and 11.9 , respectively.

\section{E. Discussion}

The experiments show that it is possible to model the solar heating system with a black-box identification method, and a small number of data points. The parameters can be made to converge using as little as 150 samples by applying multiple scans. Excluding some of the parameters may improve the model performance while the structure becomes less complex, and thus even more suitable for controller design. As an extension to this work it would be interesting to study different applications for the model, like fault detection. The fact that the model adapts fast, cf. Fig. 3, implies that it could be used for on-line tracking of the model, and optimal control based on e.g. weather forecasts.

The software implementation of the algorithm that was used for the identification experiments 
is described in [14], and can be downloaded from http://www.it.uu.se/research/reports.

\section{Conclusions}

The identification experiments performed in this paper show that the dynamics of a small scale solar heating system can be identified with a recursive black-box model even for a very small amount of data, using multiple scans. The simple model structure and the general approach in addition to the small amount of data required opens up for e.g. improved controller design and thereby a more efficient utilization of the solar energy. Such improved control methods would improve the economic benefits of solar heating, thereby contributing to an extended use of environmentally friendly energy sources.

The identification method used was an RPEM based on a restricted black-box state space model. The initial model was reduced by removal of parameters, which, for certain parameter combinations, significantly improved the model performance. The best model obtained was also compared to an energy balance based least squares method. The models proved to have comparable performance, the MSE of the least squares model being slightly higher. Some differences of the models that are worth noting are that the RPEM method studied in this paper is an OE method, which is normally less sensitive to unmodeled system dynamics compared to least squares methods. An other thing worth mentioning is that the results from the RPEM can be interpreted directly in terms of the physical meaning of the continuous time parameters.

Interesting topics for further research could be to study optimal controller design, possibly based on weather fore- casts. Model based fault detection would also be of interest. The model could also be extended to including energy consumption.

\section{REFERENCES}

[1] M. Barão, J. M. Lemos, and R. N. Silva, "Reduced complexity adaptive nonlinear control of a distributed collector solar field," Journal of Process Control, vol. 12, pp. 131-141, 2002.

[2] T. A. Johansen and C. Storaa, "Energy-based control of a distributed solar collector field," Automatica, vol. 38, pp. 1191-1199, 2002.

[3] M. Berenguel, M. R. Arahal, and E. F. Camacho, "Modeling of the free response of a solar plant for predictive control," Control engineering practice, vol. 6, pp. 1257-1266, 1998.

[4] K. J. Åström and R. D. Bell, "Drum boiler dynamics," Automatica, vol. 36 , pp. 363-378, 2000

[5] J. Funkqvist, "On modeling and identification of a continuous pulp digester," in Proc. SYSID 1994, Copenhagen, Denmark, 1994.

[6] F. De Bruyne, B. D. O. Anderson, and I. D. Landau, "Recursive identification of nonlinear plants operating in closed loop using kernel representations," Automatica, vol. 38, pp. 2021-2027, 2002.

[7] L. Ekstam and T. Smed, "Parameter estimation in dynamic systems with application to power engineering," Uppsala university, Uppsala, Sweden, Technical licentiate thesis UPTEC 8747R, 1987.

[8] T. Bohlin, "A case study of grey box identification," Automatica, vol. 30, pp. 307-318, 1994.

[9] T. Wigren, "Recursive identification based on nonlinear state space models applied to drum-boiler dynamics with nonlinear output equations," in Proceedings of ACC 2005, Portland, Oregon, U.S.A, 2005.

[10] _ - "Scaling of the sampling period in nonlinear system identification," in Proceedings of ACC 2005, Portland, Oregon, U.S.A, 2005.

[11] L. Ljung, 2003, personal communication.

[12] L. Ljung and T. Glad, Modellbygge och simulering, 2nd ed. Lund: Studentlitteratur, 2004, (In Swedish).

[13] L. Ljung and T. Söderström, Theory and practice of recursive identification. Cambridge, MA: M.I.T. Press, 1983.

[14] T. Wigren, "A matlab software package for recursive identification and scaling using a structured nonlinear black box model," Systems and Control, Department of Information Technology, Uppsala University, Uppsala, Tech. Rep. 2005-002, 2005. 